Review Article

\title{
Mechanism, Clinical Significance, and Treatment Strategy of Warburg Effect in Hepatocellular Carcinoma
}

\author{
Hui Chen, ${ }_{1}^{1}$ Qing Wu, ${ }^{2}$ Liu Peng, ${ }^{1}$ Ting Cao, ${ }^{1}$ Man-Ling Deng, ${ }^{1}$ Yi-Wen Liu, ${ }^{1}$ Jia Huang, \\ Yang $\mathrm{Hu},{ }^{1}$ Nian Fu, ${ }^{1}$ Ke-Bing Zhou, ${ }^{1}$ Mei-Ling Yang $\odot,{ }^{3}$ and Xue-Feng Yang ${ }^{1}$ \\ ${ }^{1}$ Department of Gastroenterology, The Affiliated Nanhua Hospital, Hengyang Medical College, University of South China, Hengyang, \\ 421002 Hunan Province, China \\ ${ }^{2}$ Department of General Practice, The Affiliated Nanhua Hospital, Hengyang Medical College, University of South China, Hengyang, \\ 421002 Hunan Province, China \\ ${ }^{3}$ Department of Oncology, The Affiliated Nanhua Hospital, Hengyang Medical College, University of South China, Hengyang, \\ 421002 Hunan Province, China
}

Correspondence should be addressed to Mei-Ling Yang; 1376343078@qq.com and Xue-Feng Yang; yxf009988@sina.com

Received 8 April 2021; Revised 12 April 2021; Accepted 15 April 2021; Published 28 April 2021

Academic Editor: Songwen Tan

Copyright (C) 2021 Hui Chen et al. This is an open access article distributed under the Creative Commons Attribution License, which permits unrestricted use, distribution, and reproduction in any medium, provided the original work is properly cited.

\begin{abstract}
Hepatocellular carcinoma (HCC) is a primary malignancy of the liver and the third leading cause of cancer death worldwide. The incidence of HCC accounts for more than $90 \%$ of primary HCC. Like most solid malignancies, the occurrence and development of HCC are closely related to the Warburg effect. The Warburg effect of HCC is mainly manifested as increased glucose uptake by HCC cells, increased glycolysis, restricted mitochondrial oxidative phosphorylation, increased pentose phosphate pathway in HCC cells, and increased glutamine decomposition. As the contribution of glycolysis to the total ATP of tumor cells generally does not exceed $50 \%$ to $60 \%$, oxidative phosphorylation (OXPHOS) still makes a considerable contribution to the ATP of tumor cells. In some cases, there will be an anti-Warburg effect. HCC Warburg effect is closely related to HCC cell proliferation, apoptosis, immune escape, migration and invasion, chemotherapy resistance, and treatment failure. The mechanism of the Warburg effect in HCC is complex, involving the expression of stimulating the key glycolysis enzymes by hypoxia-inducible factor-1(HIF-1), the activation of oncogenes and the inactivation of tumor suppressor genes, the continuous activation of related signaling pathways, the participation of noncoding RNA, and the rate of metabolism gene mutation of enzyme. This article synthetically discusses the characteristics of glucose metabolism in HCC cells, the mechanism of Warburg effect, clinical significance, and corresponding treatment strategies and provides new perspectives for the prevention and treatment of HCC.
\end{abstract}

\section{Preface}

For most normal cells, glucose metabolism with glucose as a substrate is the main energy metabolism method. One of the main characteristics that distinguish tumor cells from normal cells is that tumor cells mainly use glycolysis of glucose to produce lactic acid for metabolic energy production, while oxidative phosphorylation (OXPHOS) is in a relatively inhibited state, this is the Warburg effect of tumor cells (Figure 1) [1]. Tumor cells can evade the normal apoptosis program through the Warburg effect and enhance the proliferation and migration ability, which is a key factor in the pathogenesis of tumors. The Warburg effect can not only occur in the hypoxic tumor microenvironment but can also occur under aerobic conditions, so it is also called "aerobic glycolysis" [2]. It is that tumor cells use a series of molecular mechanisms to weaken aerobic respiration under aerobic conditions, carry out efficient glycolysis, and then obtain a large amount of adenosine triphosphate (ATP) and create a microenvironment suitable for tumor cells to survive proliferation advantage. In addition, the Warburg effect suppresses the monitoring and lethality of $\mathrm{T}$ lymphocytes through local hypoxia, creating tumor immune escape. By inhibiting the pathways related to the Warburg effect, some antitumor drugs such as glucose and dichloroacetic acid can more effectively inhibit the proliferation advantage and immune escape 


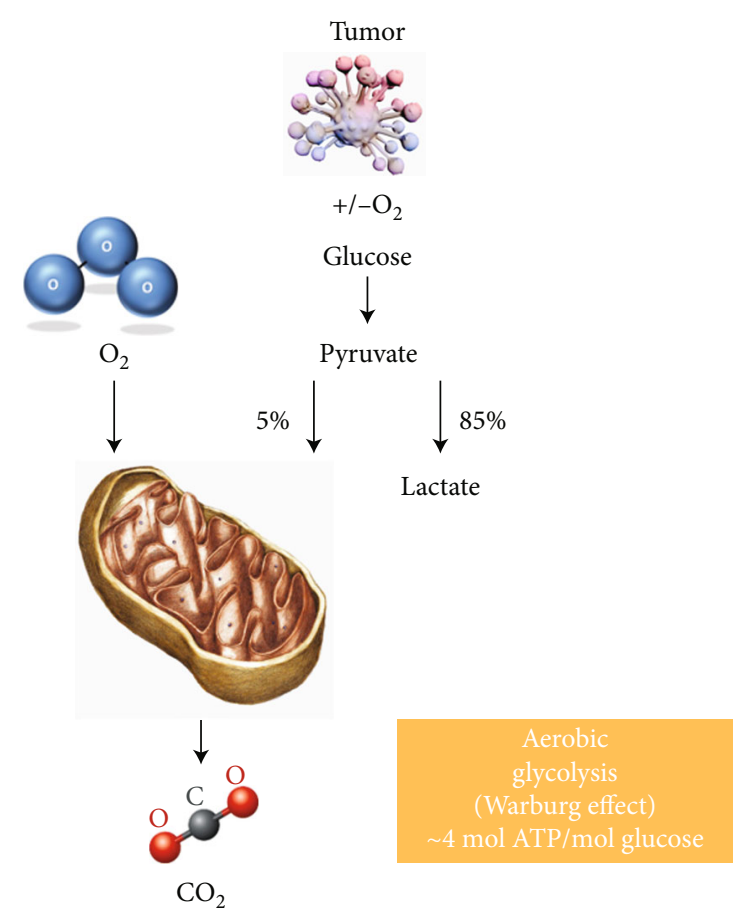

FIgURE 1: Tumor Warburg effect.

of tumor cells brought about by the Warburg effect, thereby inhibiting tumor cell growth and promoting tumor cell death. Further studies have found that the contribution of glycolysis to the total ATP of tumor cells generally does not exceed 50\% 60\% [3]; therefore, OXPHOS still makes a considerable contribution to the ATP of tumor cells. In some cases, there will be an anti-Warburg effect [4]. Hepatocellular carcinoma (HCC) is the main histological subtype of liver cancer, accounting for $90 \%$ of primary liver cancer, and is the third most common cause of cancer-related mortality worldwide [5]. Like most solid malignancies, Warburg effect plays an important role in the pathogenesis of $\operatorname{HCC}[1,6]$. This article synthetically discusses the characteristics of glucose metabolism in HCC cells, the mechanism of Warburg effect, clinical significance, and corresponding treatment strategies and provides new perspectives for the prevention of HCC and the development of targeted drugs.

\section{HCC Warburg Effect}

2.1. Increased Glucose Uptake of HCC Cells. After glucose is absorbed into the blood from the digestive tract, it needs to enter the cells for metabolism in the body. This process relies on glucose transporter (GLUT). On March 30, 2020, Boyi Gan's research group at MD Anderson Cancer Center in the United States and his collaborators published a paper in Nature Cell Biology, stating that they found that cystine ingested by the cystine transporter SLC7A11 was reduced to cysteine in the cell. The acid needs to consume a large amount of reduced nicotinamide adenine dinucleotide phosphate (NADPH). Intracellular NADPH is mainly derived from the bypass of glucose metabolism-the pentose phosphate pathway, so it is highly dependent on the supply of glucose. In the absence of glucose, the high expression of SLC7A11 in cancer cells will lead to the significant accumulation of cystine and other disulfides in the cell, the breakdown of the redox system, and the rapid cell death. It suggests that for patients with cancer cells, the lack of glucose will have a more adverse effect on the body. 14 human GLUTs (GLUT1-14) have been found, and they play a role in different tissues and cells; most of which are expressed in liver tissues [7, 8]. Compared with nontumor tissues, the expression of GLUT-1 in HCC was significantly increased $[8,9]$, suggesting that HCC cells have increased glucose uptake. Amann et al. [9] found that the expression level of GLUT1mRNA in HCC tissues and cell lines was significantly increased. Inhibiting the expression of GLUT1 in HCC cell lines can reduce glucose uptake and lactate secretion.

2.2. Enhanced Glycolysis of HCC Cells. Under normal circumstances, most cells in the human body mainly rely on the tricarboxylic acid (TCA) cycle and OXPHOS to be fully oxidize to supply cell energy under aerobic conditions; in the case of hypoxia, it mainly relies on glycolysis production capacity to supply cell energy. The productivity of glycolysis is higher than that of OXPHOS, allowing cancer cells to gain a competitive advantage. In addition to energy production, glycolysis also provides many precursor substances, which can maintain the tumor microenvironment and the stability of HIF. A few cells with extremely metabolically active, such as nerve cells and white blood cells, often use glycolysis to provide part of their energy even if they are not hypoxic. Individual cells, such as mature red blood cells, are completely dependent on glycolysis due to lack of mitochondria. Like most tumor cells, HCC cells mainly rely on glycolysis to supply cell energy, whether aerobic or anaerobic. This is the so-called Warburg Phenomenon, also known as "aerobic glycolysis," which is manifested in the increased expression and enhanced activity of the rate-limiting enzyme of glycolysis. For example, the of hexokinase (HK) increases, 6-phosphofructokinase-1 (G6P) increases, pyruvate kinase (PK) increases and activity enhance, and the metabolite lactic acid increases $[1,2,10,11]$.

2.3. Limited Mitochondrial Oxidative Phosphorylation. The aerobic oxidation of glucose is roughly divided into three stages. The first stage: glucose is decomposed into pyruvate in the cytoplasm through glycolysis. The second stage: pyruvate enters the mitochondria, oxidative decarboxylation produces acetyl CoA. This process is catalyzed by the pyruvate dehydrogenase complex. The third stage: acetyl CoA TCA cycle and OXPHOS in the mitochondria. It is generally believed that TCA is mainly regulated by isocitrate dehydrogenase (IDH) and $\alpha$-ketoglutarte dehydrogenase complex. The TCA is the final metabolic pathway of the three major nutrients of sugar, fat, and amino acids. The oxidation of glucose, fat, and amino acids in the body eventually produces acetyl CoA, which then enters TCA for decomposition. TCA itself does not produce ATP, only OXPHOS reaction to generate ATP to provide $\mathrm{NADH}^{+} \mathrm{H}^{+}$and $\mathrm{FADH} 2$. OXPHOS is coupled with ADP phosphorylation to generate ATP during the electron transport process of the respiratory 
chain. As early as 1996, Capuano et al. discovered mitochondria isolated from biopsies of human hepatocellular carcinoma exhibit a decreased rate of respiratory ATP synthesis and a decreased ATPase activity [12].

2.4. Enhancing the Pentose Phosphate Pathway of HCC Cells. The pentose phosphate pathway (PPP) is another way of oxidative decomposition of glucose besides glycolysis and TCA cycle. PPP is also called the hexose monophosphate shunt. It is a pathway through which glucose-6-phosphate is metabolized to produce NADPH and ribose-5-phosphate. Glucose6-phosphate dehydrogenase (glucose-6-phosphate dehydrogenase, G6PD) is the rate-limiting enzyme of the oxidative arm of the PPP, and its expression is significantly increased in HCC tissues. The data obtained by Kowalik MA and others from patients undergoing HCC resection or liver biopsy showed that compared with their adjacent tissues, G6PD was significantly upregulated in most HCC tissues [13]. PPP includes two stages: oxidation and nonoxidation. In the oxidation stage, glucose-6-phosphate is converted to ribulose-5-phosphate and $\mathrm{CO} 2$, and two molecules of $\mathrm{NADPH}$ are generated; in the nonoxidation stage, ribulose5-phosphoric acid is isomerized to ribose-5-phosphate or converted into two intermediate metabolites in glycolysis, fructose-6-phosphate and glyceraldehyde-3-phosphate. For tumor cells, the most important effect of PPP is produce NADPH, maintain redox homeostasis, prevent oxidative damage, and protect tumor cells.

2.5. Enhanced Decomposition of Glutamine. Glycolysis is dominant in most tumor energy metabolism, but some tumor cells still use OXPHOS as the main energy production pathway, or a mixed energy production pathway of glycolysis and OXPHOS [14]. Due to the Warburg effect, tumor cells obtain biosynthetic precursors and NADPH through the TCA cycle. The demand for biosynthetic precursors and NADPH has increased dramatically. In order to meet the needs, tumor cells often rely on the decomposition of glutamine to maintain the TCA cycle and provide biosynthetic precursors and NADPH $[15,16]$. It was discovered a long time ago that in the human HCC cell line HepG2 cells, when glutamine is sufficient, the promoter of its transporter ASCT2 is activated, which increases the expression of ASCT2 [17].

\section{HCC Glucose Metabolism Reverse Warburg Effect}

Some tumors have two-compartment tumor metabolism or metabolic coupling. In the biphasic model of tumor metabolism, tumor cells induce the surrounding stromal fibroblasts to produce the Warburg effect, which makes the fibroblasts differentiate into myofibroblasts. Through aerobic glycolysis, the myofibroblasts produce a large amount of energy metabolism such as lactic acid and pyruvate. However, when glucose is metabolized to lactic acid, only 2 ATP is produced per glucose molecule, and after a glucose molecule is completely oxidized, oxidative phosphorylation can produce up to 36 ATP. However, tumor cells preferentially ferment glucose even when oxygen is sufficient. This is because no matter how much stimulation, the aerobic glycolysis cells have a high ratio of $\mathrm{ATP} / \mathrm{ADP}$ and $\mathrm{NADH} / \mathrm{NAD}+$. In addition, even small changes in the ATP/ADP ratio can affect its growth. Cells with insufficient ATP usually undergo apoptosis. When the ability of normal proliferating cells to produce ATP from glucose metabolism is impaired, they will undergo cell cycle arrest and reactivate catabolism. At the same time, there are signaling pathways to sense energy status. The product is then transported to tumor cells, enters the tumor cell mitochondria, provides fuel for the TCA cycle, and generates a large amount of ATP through oxidative phosphorylation, which promotes tumor cell proliferation and resists apoptosis $[18,19]$. Whether HCC cells provide fuel for the TCA cycle through two-compartment tumor metabolism has not been reported in the literature.

\subsection{Mechanism of Warburg Effect in HCC}

\subsubsection{The Role of Hypoxia-Inducible Factor-1 (HIF-1)}

(1) HIF1 Upregulates the Expression of GLUT1 in HCC Cells. Due to the rapid growth of tumor cells, the formation of new blood vessels is relatively lagging, and most of the new blood vessels are malformed and cannot adequately supply nutrients and oxygen. Therefore, there is hypoxia or hypoxia in most tumor tissues. Hypoxia-induced HIF1 can upregulate the expression of GLUT1 on the surface of HCC cells and increase glucose uptake [20]. Inhibiting the expression of hypoxia-inducible factor 1 alpha, the expression of GLUT1 in HCC cells was significantly reduced, and the uptake of glucose was reduced [21].

(2) HIF1 Stimulates the Expression of Glycolytic RateLimiting Enzymes. Zhuonan et al. found that while the expression of HIF-1 in liver tissue increased in low temperature environment, the levels of $\mathrm{HK}-2$ and pyruvate dehydrogenase kinase (PDK-1) increased [22]. Golinska et al. investigated the consequences of HIF-1 deficiency in mice, using the well-established Hepa-1 wild-type (WT) and HIF$1 \beta$-deficient (c4) model, found that HIF-1 $\beta$-deficient Hepa1 c4 tumours grew significantly more slowly than WT tumours, and (as expected) showed significantly lower expression of many glycolytic enzymes [23]. Mathupala et al. found the hepatoma cells transfected type II hexokinase gene promoter to hypoxic conditions activate the type II hexokinase promoter almost 3-fold, a value that approaches 7 -fold in the presence of glucose [10].

(3) HIF1 Stimulates the Expression of Pyruvate Dehydrogenase Kinase 1. Increased expression of HIF-1 can be found in almost all HCC tissues [24]. HIF-1 is particularly crucial for shifting the metabolic program of cancer cells from oxidative phosphorylation to glycolysis [25]. HIF-1 can stimulate the expression of PDK1; PDK1 can phosphorylate the pyruvate dehydrogenase complex, inactivate the latter and then prevent acetone acid enters the TCA cycle [26].

(4) HIF1 Stimulates the Expression of a Less Active Complex I Subunit. Hypoxia results in an inefficient transfer of electrons 
TABLE 1: The effects and targets of hypoxia-inducible factor-1 (HIF-1) on glucose metabolism of HCC cells.

\begin{tabular}{|c|c|c|c|}
\hline Metabolism type & Target & Effects & Reference \\
\hline Glucose uptake & GLUT1 & Upregulated expression, enhanced activity & {$[21,22]$} \\
\hline \multirow{3}{*}{ Aerobic glycolysis } & 6-phosphofructokinase-1 & Upregulated expression & [23] \\
\hline & Hexokinase & Upregulated expression & [23-25] \\
\hline & Pyruvate kinase & Upregulated expression & {$[25]$} \\
\hline \multirow{5}{*}{$\begin{array}{l}\text { Tricarboxylic acid cycle and } \\
\text { oxidative phosphorylation }\end{array}$} & Pyruvate dehydrogenase complex & $\begin{array}{c}\text { Upregulate the expression of pyruvate } \\
\text { dehydrogenase kinase } 1\end{array}$ & [27] \\
\hline & Isocitrate dehydrogenase, IDH & & \\
\hline & $\alpha$-Ketoglutarte dehydrogenase complex & & \\
\hline & Complex I-IV & Upregulate NDUFA4L2 expression & [28] \\
\hline & ATP synthase & & \\
\hline Pentose phosphate pathway & Glucose-6-phosphate dehydrogenase, G6PD & & \\
\hline \multirow{2}{*}{ Glutamine metabolism } & ASCT2 & & \\
\hline & Glutaminase & & \\
\hline
\end{tabular}

during oxidative phosphorylation leading to increased oxidative stress. NDUFA4L2 is a less active subunit of complex I in the oxidative phosphorylation electron transport chain, and it is also a direct transcriptional target of HIF [27]. Lai et al. found that HIF inhibitor, digoxin, profoundly inhibited growth of tumors that expressed high level of NDUFA4L2 in orthotopic model [27].

In summary, HIF-1 plays an important role in the metabolic reprogramming of HCC. The effect of HIF-1 on the glucose metabolism of HCC cells and its targets are shown in Table 1.

\subsubsection{Activation of Oncogenes and Inactivation of Tumor Suppressor Genes}

(1) Upregulation of GLUT1 Expression in HCC Cells. In HCC cells, the activated protooncogenes mainly include c-myc and $\mathrm{K}$-Ras. The mutations of the protooncogenes c-myc and KRas can upregulate the expression of glucose transporters on the surface of HCC cells, thereby increasing glucose uptake. Glypican-3 enhances the glycolysis of HCC cells by upregulating the glycolysis genes of GLUT1, HK2, and LDH-A $[28,29]$. This may be one of the reasons for the enhanced glycolysis in HCC cells.

(2) Stimulating the Expression of Glycolytic Rate-Limiting Enzymes. C-myc and K-Ras gene mutations can not only upregulate the expression of GLUT1 in HCC cells but also stimulate the expression of glycolytic rate-limiting enzymes, such as HK, G6P, and PK, thereby increasing the level of glycolysis $[28,29]$. p53 is a major tumor suppressor gene; wildtype p53 can suppress tumour development by multiple pathways, mutation of TP53, and the resultant inactivation of p53 allow evasion of tumour cell death and rapid tumour progression [30]. p53 in half mutations occur in human cancers (in half of all human cancers) [31], but the wild-type p53 (WTp53) is often retained in HCC and suppressing pyruvate-driven oxidative phosphorylation by inducing
PUMA [30]. The role of p53 in tumors is conflicting, and further research is needed.

(3) Mitochondrial Dysfunction in HCC Cells. K-Ras gene mutation can cause mitochondrial dysfunction in HCC cells, which in turn weakens the level of oxidative phosphorylation of glucose metabolism, forcing HCC cells to change their energy metabolism from oxidative phosphorylation to aerobic sugars. Fermentation is the main thing $[28,29]$.

\subsubsection{Continuous Activation of Related Signal Pathways}

(1) Promote Glycolysis of Tumor Cells. Take the $\mathrm{PI} 3 \mathrm{~K} / \mathrm{AKT} / \mathrm{mTOR}$ signaling pathway as an example. Their continuous activation can promote glycolysis of tumor cells. In the pathway, AKT is a serine/threonine kinase whose expression is significantly enhanced in HCC tissues [32], which can induce the expression of HK II and the activation and phosphorylation of sphosphofructokinase-2 (PFK2) to enhance glycolysis of HCC cells [32]. Combretastatin A4 phosphate (CA4P) can also reduce HIF-1 $\alpha$ through $\mathrm{PI} 3 \mathrm{~K} / \mathrm{AKT} / \mathrm{mTOR}$ signaling pathway and synergistically inhibit HCC [33]. Akt can also use mTOR-mediated expression of glycolytic enzymes through the activation of HIF- $1 \alpha$, $\mathrm{NF} \kappa \mathrm{B}$, and c-Myc [34].

\subsubsection{Noncoding RNA Is Involved in Regulation}

(1) Noncoding RNA Regulates GLUT1 to Participate in Glucose Uptake of Hepatoma Cells. LINC01638 lncRNA [35], lncRNA HOTAIR [36], miR-181a-5p [37], and MiR-505 [38] increase the uptake of glucose by cancer cells by upregulating the expression of GLUT1 on the surface of HCC cells and promote the proliferation of hepatocellular carcinoma cells; lncRNA SLC2A1-AS1 [39], miR-342-3p [40], miR455-5p [41], and miR-328-3p [42] can reduce the expression of GLUT1 on the surface of HCC cells. Inhibiting the uptake of glucose by cancer cells inhibits the proliferation of hepatocellular carcinoma cells. 
TABLE 2: The effects and targets of noncoding RNA on glucose metabolism of HCC cells.

\begin{tabular}{|c|c|c|c|}
\hline Metabolism type & Target & Effects & Noncoding RNAs \\
\hline \multirow{2}{*}{ Glucose uptake } & \multirow{2}{*}{ GLUT1 } & Up & $\begin{array}{c}\text { LINC01638 lncRNA [34] } \\
\text { IncRNA HOTAIR [35] } \\
\text { miR-181a-5p [36] } \\
\text { MiR-505 [37] }\end{array}$ \\
\hline & & Down & $\begin{array}{c}\text { IncRNA SLC2A1-AS1 [38] } \\
\text { miR-342-3p [39] } \\
\text { miR-455-5p [40] } \\
\text { miR-328-3p [41] }\end{array}$ \\
\hline \multirow{5}{*}{ Aerobic glycolysis } & 6-Phosphofructokinase-1 & $\begin{array}{c}\text { Up } \\
\text { Down }\end{array}$ & \\
\hline & \multirow[t]{2}{*}{ Hexokinase } & Up & miR-181a-5p [36] \\
\hline & & $\mathrm{Up}$ & $\begin{array}{l}\operatorname{miR}-4417[43] \\
\text { circMAT2B [44] }\end{array}$ \\
\hline & Pyruvate kinase & Down & $\begin{array}{c}\text { LncRNA WPDC2p [45] } \\
\text { miR-199a [46] } \\
\text { miR-491-5p [47] } \\
\text { miR-122 [48] }\end{array}$ \\
\hline & LDH & $\begin{array}{c}\text { Up } \\
\text { Down }\end{array}$ & miR-181a-5p [36] \\
\hline \multirow{5}{*}{$\begin{array}{l}\text { Tricarboxylic acid cycle and oxidative } \\
\text { phosphorylation }\end{array}$} & Pyruvate dehydrogenase complex & $\begin{array}{c}\text { Up } \\
\text { Down }\end{array}$ & \\
\hline & Isocitrate dehydrogenase, IDH & $\begin{array}{c}\text { Up } \\
\text { Down }\end{array}$ & \\
\hline & $\alpha$-Ketoglutarte dehydrogenase complex & $\begin{array}{c}\text { Up } \\
\text { Down }\end{array}$ & \\
\hline & Complex I-IV & $\mathrm{Up}$ & lncRNA MALAT1 [49] \\
\hline & ATP synthase & Down & \\
\hline Pentose phosphate pathway & Glucose-6-phosphate dehydrogenase,G6PD & $\begin{array}{c}\text { Up } \\
\text { Down }\end{array}$ & \\
\hline \multirow[t]{2}{*}{ Glutamine metabolism } & ASCT2 & $\begin{array}{c}\text { Up } \\
\text { Down }\end{array}$ & \\
\hline & Glutaminase & Up & \\
\hline
\end{tabular}

(2) Noncoding RNA Is Involved in Glycolysis of HCC. Various noncoding RNAs (ncRNAs) including microRNAs (miRNAs), long noncoding (lncRNAs), and circular RNAs (circRNAs) have recently been proved to play potential roles in glycolysis in hepatocellular carcinoma [43]. For example, miR-4417 [44] and circMAT2B [45] can activate PKM2 expression, thereby promoting glycolysis in HCC, while LncRNA WPDC2p [46], miR-199a [47], MiR-491$5 p$ [48], and miR-122 [49] can inhibit the expression of PKM2.

(3) Noncoding RNA Is Involved in TCA and OXPHOS. The mitochondria of HCC cells contain a large amount of lncRNA metastasis-associated lung adenocarcinoma transcript 1 (MLAT1). MALAT1 knockdown induced alterations in the $\mathrm{CpG}$ methylation of mtDNA, and in mitochondrial transcriptomes, at the same time, HCC cells have changes in mitochondrial structure, low oxidative phosphorylation (oxphos), and decreased ATP production [50].
In short, noncoding RNA can affect HCC metabolism through multiple pathways, and its effects on HCC cell carbohydrate metabolism and targets are shown in Table 2.

3.1.5. Mutations in Rate-Limiting Enzyme Genes. According to reports, some specific types of HCC IDH1 may show a higher mutation rate. For example, Jung et al. detected 48 cases of HCC (including 20 cases of clear cell type and 13 cases of pseudoglandular pattern) IDH1, with a mutation rate of 10.4\%; all of which were clear cell type HCC [51]. IDH is one of the rate-limiting enzymes of the TCA cycle, responsible for the oxidative decarboxylation of isocitrate into $\alpha$-ketoglutarate and $\mathrm{NADH}^{+} \mathrm{H}^{+}$. IDH mutation changes the active site of the enzyme and endows the enzyme with new catalytic activity, using $\alpha$-ketoglutar-ate $(\alpha-\mathrm{KG})$ to produce high levels of R-2-hydrox-yglutarate (R-2-HG); the molecular structure of $2-\mathrm{HG}$ and $\alpha-\mathrm{KG}$ is very similar [52], and it is speculated that 2-HG may participate in OXPHOS by inhibiting $\alpha$-KG through competition. 
3.1.6. Glycolysis Inhibits Oxidative Glycolysis. Domenis et al. reported that the well-differentiated HCC cell line HepG2 OXPHOS system is rarely damaged [53]. If glycolysis is inhibited, such as silencing the expression of hexokinase- 2 in HCC cells, the flow of glucose to pyruvate and lactic acid is inhibited, but the flow of tricarboxylic acid is maintained [54]. As we all know, the metabolic fate of pyruvate produced by glycolysis is determined by the route of $\mathrm{NADPH}+\mathrm{H}+$. When aerobic $\mathrm{NADPH}+\mathrm{H}+$ enters the mitochondria for oxidation, pyruvate undergoes aerobic oxidation without producing lactic acid. NADPH $+\mathrm{H}+$ cannot be oxidized under hypoxia; pyruvic acid acts as a hydrogen acceptor to generate lactic acid. In most cases, HCC cells are in a hypoxic microenvironment, $\mathrm{NADPH}+\mathrm{H}+$ cannot be oxidized, pyruvate produced by glycolysis can only be used as a hydrogen acceptor to produce lactic acid, and oxidative phosphorylation is restricted.

3.1.7. Mitochondria Are Damaged. The "Warburg effect" illustrates that tumor cells tend to choose aerobic glycolysis over OXPHOS, as does HCC cells. In the past, as represented by Warburg, it was believed that tumor cells mainly used aerobic glycolysis for energy because mitochondria were damaged, and oxidative phosphorylation dysfunction occurred [1]. It is now believed that the reprogramming of tumor cell metabolic pathways is not caused by simple mitochondrial dysfunction [55].

\section{The Clinical Significance of Warburg Effect of HCC Glucose Metabolism}

\subsection{The Clinical Significance of the Warburg Effect of HCC Glucose Metabolism}

4.1.1. Warburg Effect Promotes the Proliferation of HCC Cells. The Warburg effect is a metabolic hallmark of cancer. Tumor cells rapidly adjust their energy source to glycolysis in order to efficiently proliferate in a hypoxic environment [56]. The main physiological significance of glycolysis. It is to provide energy quickly. The ATP production rate of glycolysis may be 100 times faster than that of OXPHOS [57]. The production rate of ATP produced by glycolysis increases, giving cancer cells a selective growth advantage. Inhibition of the expression of key enzymes of glycolysis and tumor cell proliferation is inhibited. For example, when HK2 expression is silenced, glucose flux to pyruvate and lactate of HCC cells is inhibited, and the proliferation and death of HCC cells increase at the same time [58].

4.1.2. Warburg Effect Inhibits Apoptosis of HCC Cells. Pyruvate dehydrogenase $\mathrm{E} 1 \alpha$ subunit (PDHA1) is one of the main factors for metabolic switch from OXPHOS to aerobic glycolysis and has been suggested to be closely associated with tumorigenesis [59]. Upregulated PDHA1 gene expression in HCC cell line SMMC-7721 and HepG2 can inhibit Warburg effect and enhanced mitochondria-mediated apoptosis pathway [59].

4.1.3. Warburg Effect Promotes Tumor Cell Metastasis. Aerobic glycolysis can produce a large amount of lactic acid and creat an acidic microenvironment, which is conducive to tumor cell invasion and metastasis [43]. Todisco et al. [17] found that increased G6PD expression is related to HCC metastasis and poor prognosis, while decreased g6pd expression inhibits the proliferation, migration, and invasion of HCC cell lines.

4.1.4. Warburg Effect Ultimately Leads to Tumor Immune Escape. The enhanced of tumor aerobic glycolysis is accompanied by the production and extracellular transport of a large amount of glycolysis end product in tumor cells, which leads to lactic acid in the tumor microenvironment accumulation and acidic microenvironment. The high lactate titer and acidic microenvironment outside the cell can directly hinder the extracellular transport of CTL and NK cells, thereby directly affecting the proliferation and cytokine secretion of CTL and NK cells, resulting in impaired killing function [60]. On the other hand, the acid degradation of IFN- $\gamma$ weakens the differentiation of initial T cells into tumor suppressor type 1 helper T cells (Th1) and promotes them to differentiate into type 2 helper T cells (Th2) [61] and promote the proliferation of myeloid-derived suppressor cells (MDSC) in the tumor, and ultimately lead to the immune escape of the tumor through a variety of mechanisms.

\subsection{The Clinical Significance of the Reverse Warburg Effect of HCC Glucose Metabolism}

4.2.1. Promote Tumor Cell Proliferation and Resist Apoptosis. Martinez-Outschoorn et al. [62] found that some tumors have a two-compartment tumor metabolism, which is called the reverse Warburg effect or metabolic coupling. In the biphasic model of tumor metabolism, epithelial tumor cells induce the surrounding stromal fibroblasts to produce the Warburg effect, which makes the fibroblasts differentiate into myofibroblasts, and the myofibroblasts produce large amounts of energy such as lactic acid and pyruvate through aerobic glycolysis. The metabolites are then transported to epithelial tumor cells, enter the tumor cell mitochondria, provide fuel for the TCA cycle, and generate large amounts of ATP through OXPHOS to promote tumor cell proliferation and resist apoptosis $[63,64]$.

4.2.2. It May Be the Cause of Chemotherapy Resistance and Treatment Failure. The biphasic metabolism of tumor may be the cause of chemotherapy resistance and treatment failure, and it may also explain the contradictory phenomenon of high mitochondrial respiration and low glycolysis rate in some tumor cells [65]. This pattern indicates that there is a host-parasite relationship between tumor cells and surrounding fibroblasts or metabolic coupling [7]. The metabolic coupling includes two levels: (i) the metabolic coupling of fibroblasts and tumor cells and (ii) the coupling of aerobic glycolysis and OXPHOS in tumor cells.

\section{Treatment Strategies for HCC Based on Positive and Negative Warburg Effect}

5.1. Before Treatment with Energy Metabolism Drugs, It Is Necessary to Evaluate the Metabolic Characteristics of HCC Patients. Due to the difference in cell origin and 
differentiation of tumors, not all tumor cells exhibit glycolysis, and the contribution of glycolysis to the total ATP of tumor cells ranges from $1 \%$ to $64 \%$ [66]. HCC is a highly heterogeneous cancer, both at the molecular and histological level [67]. According to the WHO classification standards, it is divided into highly differentiated, moderately differentiated, and poorly differentiated HCC. Poorly differentiated, undifferentiated, and fast-growing tumors are generally glycolysis-based, and OXPHOS is inhibited. For example, the undifferentiated HCC cell line JHH-6XPHOS system is significantly damaged, and its metabolism is mainly glycolysis [68].

5.2. According to the Patient's Metabolic Characteristics, Select the Appropriate Treatment Drug. HCC cells, like most tumors, have different cellular energy metabolism, but in general, they are still dominated by glycolytic phenotypes and rely on the consumption of glutamine. Studies have found that even if the tumor tissue is rich in oxygen, its glycolysis is still in the production capacity, but oxidative phosphorylation is relatively inhibited [6]. The specific strategy is as follows.

5.2.1. Reduce Glucose Transport into Cells. The GLUT1 gene is a key rate-limiting factor encoding glucose transport to cancer cells. Shang et al. [69] found that using siRNA to inhibit the expression of GLUT1, HCC cells are reducing glucose. At the same time of uptake and secretion of lactic acid, the growth and migration potential of HCC cells is weakened [69].

5.2.2. Inhibition of Glycolysis. Hexokinase is the first ratelimiting enzyme in the glycolysis pathway. Hexokinase catalyzes the phosphorylation of glucose into glucose 6phosphate. Glucose 6-phosphate can be used for glycolysis or pentose phosphate pathway. DeWaal et al. reported that silencing HK2 expression, HCC cell glucose flux to pyruvate and lactate is inhibited, while HCC cell proliferation decreased and death increased, knockout HK2 in mice can reduce proliferation and the incidence of tumors induced by diethylnitrosamine (DEN) [11].

5.2.3. Inhibition of Decomposition of Glutamine. Glutamine is the amide of glutamic acid. L-glutamine is the amino acid encoded in protein synthesis. It is a nonessential amino acid in mammals and can be converted from glucose in the body. Lee et al. reported that mitochondrial dependence on glutamine can drive the chemotherapy resistance of HCC stem cells [70]. The high level of glutamine in the blood provides a ready source of carbon and nitrogen to support the biosynthesis, energy metabolism, and cell homeostasis of cancer cells and promote tumor growth. Glutamine is transported into the cell by the transporter SLC1A5 (the solute carrier family 1 neutral amino acid transporter member 5) in the cell. Under nutrient-deficient conditions, cancer cells can obtain glutamine by breaking down macromolecules. Excessive activation of the oncogene RAS can promote pinocytosis. Cancer cells remove extracellular proteins and degrade them into amino acids including glutamine to provide nutrients for cancer cells.
5.2.4. Inhibition of the Pentose Phosphate Pathway. The pentose phosphate pathway is a branch of glycolysis and a bypass pathway of glucose metabolism, producing NADPH and pentose. NADPH is a hydrogen donor for various anabolism in the body, and pentose is a nucleoside and is necessary for rapid cell proliferation. The same study reported that G6PD silencing significantly inhibited HepG2 cell line invasion [71]. In vivo studies reinforced the relevance of G6PD in HCC progression, as G6PD suppression inhibited tumor growth in Huh7 orthotopic tumor and mouse xenograft models [72].

5.2.5. Promote Oxidative Phosphorylation. Pyruvate dehydrogenase $\mathrm{E} 1 \alpha$ subunit (PDHA1) is one of the main factors for the metabolic switch from OXPHOS to aerobic glycolysis and has been suggested to be closely associated with tumorigenesis. Sun etc. observed that the PDHAl protein was reduced in HCC specimens by immunohistochemistry and Western blot, which was significantly associated with poor overall survival [73]. At the same time, mitochondrial OXPHOS was enhanced accompanied with higher ATP. Sun et al. also found that apoptosis was promoted, and intrinsic pathway proteins were increased in PDHA1overexpressing cells.

5.3. Combination Medication. Due to the high metabolic adaptability of tumor cells, when any metabolic pathway encounters obstacles, tumor cells will automatically switch or activate other pathways to avoid stress damage. Therefore, tumor metabolic regulation therapy should jointly block or regulate multiple metabolisms. The biphasic metabolism of tumors may be the cause of chemotherapy resistance and treatment failure [74]. For HCC chemotherapy resistance and treatment failure, multisite and multipath combination medication should be considered.

\section{Summary}

HCC is a primary liver cancer with high mortality, and its pathogenesis is related to the Warburg effect. The Warburg effect of HCC is mainly manifested in the increased glucose uptake of HCC cells, increased glycolysis, restricted mitochondrial oxidative phosphorylation, increased pentose phosphate pathway in HCC cells, and increased glutamine decomposition. In some cases, there will be a reverse Warburg effect. HCC Warburg effect and reverse Warburg effect are closely related to HCC cell proliferation, apoptosis, immune escape, migration and invasion, chemotherapy resistance, and treatment failure. Therefore, subsequent indepth discussions on the Warburg effect mechanism of liver cancer cells and the corresponding clinical significance can provide a new way for the prevention of liver cancer and the development of targeted drugs.

\section{Data Availability}

The datasets used and/or analyzed during the current study are available from the corresponding author on reasonable request. 


\section{Conflicts of Interest}

The authors declare no conflict of interest, financial or otherwise.

\section{Authors' Contributions}

Hui Chen and Qing Wu equally contributed to this work. Hui Chen, Qing Wu, Liu Peng, Ting Cao, Man-Ling Deng, Yi-Wen Liu, Jia Huang, Yang Hu, Nian Fu, Ke-Bing Zhou, Mei Ling Yang, and Xue-Feng Yang all contributed to the literature review and the writing of this manuscript. Hui Chen and Qing $\mathrm{Wu}$ are all co-first authors.

\section{Acknowledgments}

This work was supported by the Scientific Research Project of Hunan Provincial Health and Family Planning Commission (No. A2017015); the Natural Science Foundation of Hunan Province, China, (No. 2016JJ5010); and the National Natural Science Foundation of China (No. 81373465).

\section{References}

[1] O. Warburg, "On the origin of cancer cells," Science, vol. 123, no. 3191, pp. 309-314, 1956.

[2] X. L. Zu and M. Guppy, "Cancer metabolism: facts, fantasy, and fiction," Biochemical and Biophysical Research Communications, vol. 313, no. 3, pp. 459-465, 2004.

[3] W. H. Koppenol, P. L. Bounds, and C. V. Dang, "Otto Warburg's contributions to current concepts of cancer metabolism," Nature Reviews. Cancer, vol. 11, no. 5, pp. 325-337, 2011.

[4] M. Lee and J. H. Yoon, "Metabolic interplay between glycolysis and mitochondrial oxidation: the reverse Warburg effect and its therapeutic implication," World Journal of Biological Chemistry, vol. 6, no. 3, pp. 148-161, 2015.

[5] F. Bray, J. Ferlay, I. Soerjomataram, R. L. Siegel, L. A. Torre, and A. Jemal, "Global cancer statistics 2018: GLOBOCAN estimates of incidence and mortality worldwide for 36 cancers in 185 countries," CA: a Cancer Journal for Clinicians, vol. 68, no. 6, pp. 394-424, 2018.

[6] J. Feng, J. Li, L. Wu et al., "Emerging roles and the regulation of aerobic glycolysis in hepatocellular carcinoma," Journal of Experimental \& Clinical Cancer Research, vol. 39, no. 1, p. $126,2020$.

[7] S. Karim, D. H. Adams, and P. F. Lalor, "Hepatic expression and cellular distribution of the glucose transporter family," World journal of gastroenterology, vol. 18, no. 46, pp. 67716781, 2012.

[8] T. Aboushousha, S. Mamdouh, H. Hamdy et al., "Immunohistochemical and biochemical expression patterns of TTF-1, RAGE, GLUT-1 and SOX2 in HCV-associated hepatocellular carcinomas," Asian Pacific Journal of Cancer Prevention, vol. 19, no. 1, pp. 219-227, 2018.

[9] T. Amann, U. Maegdefrau, A. Hartmann et al., “_GLUT1_ Expression Is Increased in Hepatocellular Carcinoma and Promotes Tumorigenesis," The American Journal of Pathology, vol. 174, no. 4, pp. 1544-1552, 2009.
[10] S. P. Mathupala, A. Rempel, and P. L. Pedersen, "Glucose Catabolism in Cancer Cells:," The Journal of Biological Chemistry, vol. 276, no. 46, pp. 43407-43412, 2001.

[11] W. Gao, J. Sun, F. Wang et al., "Deoxyelephantopin suppresses hepatic stellate cells activation associated with inhibition of aerobic glycolysis via hedgehog pathway," Biochemical and Biophysical Research Communications, vol. 516, no. 4, pp. 1222-1228, 2019.

[12] F. Capuano, D. Varone, N. D'Eri et al., "Oxidative phosphorylation and $\mathrm{F}(\mathrm{O}) \mathrm{F}(1)$ ATP synthase activity of human hepatocellular carcinoma," Biochemistry and Molecular Biology International, vol. 38, no. 5, pp. 1013-1022, 1996.

[13] M. A. Kowalik, A. Columbano, and A. Perra, "Emerging role of the pentose phosphate pathway in hepatocellular carcinoma," Frontiers in oncology, vol. 7, p. 87, 2017.

[14] D. Chandra and K. K. Singh, "Genetic insights into OXPHOS defect and its role in cancer," Biochimica Biophysica Acta (BBA)-Bioenergetics, vol. 1807, no. 6, pp. 620-625, 2011.

[15] M. H. Gao, P. Monian, N. Quadri, R. Ramasamy, and X. Jiang, "Glutaminolysis and transferrin regulate ferroptosis," Molecular Cell, vol. 59, no. 2, pp. 298-308, 2015.

[16] V. H. Villar, F. Merhi, M. Djavaheri-Mergny, and R. V. Durán, "Glutaminolysis and autophagy in cancer," Autophagy, vol. 11, no. 8, pp. 1198-1208, 2015.

[17] C. I. Bungard and J. D. McGivan, "Glutamine availability up-regulates expression of the amino acid transporter protein ASCT2 in HepG2 cells and stimulates the ASCT2 promoter," The Biochemical Journal, vol. 382, no. 1, pp. 27-32, 2004.

[18] S. Todisco, P. Convertini, V. Iacobazzi, and V. Infantino, "TCA cycle rewiring as emerging metabolic signature of hepatocellular carcinoma," Cancers (Basel), vol. 12, no. 1, p. 68, 2019.

[19] M. A. C. Déry, M. D. Michaud, and D. E. Richard, "Hypoxiainducible factor 1: regulation by hypoxic and non-hypoxic activators," The International Journal of Biochemistry \& Cell Biology, vol. 37, no. 3, pp. 535-540, 2005.

[20] X. Hongping, C. Jianxiang, G. Hengjun et al., "Hypoxia-inducible factors in physiology and medicine," Cell, vol. 148, no. 3, pp. 399-408, 2012.

[21] T. Mizuno, M. Nagao, Y. Yamada et al., "Small interfering RNA expression vector targeting hypoxia-inducible factor 1 alpha inhibits tumor growth in hepatobiliary and pancreatic cancers," Cancer Gene Therapy, vol. 13, no. 2, pp. 131-140, 2006.

[22] Z. Zhuonan, G. Sen, J. Zhipeng et al., "Hypoxia preconditioning induced HIF- $1 \alpha$ promotes glucose metabolism and protects mitochondria in liver I/R injury," Clinics and Research in Hepatology and Gastroenterology, vol. 39, no. 5, pp. 610619, 2015.

[23] M. Golinska, H. Troy, Y. L. Chung et al., "Adaptation to HIF-1 deficiency by upregulation of the AMP/ATP ratio and phosphofructokinase activation in hepatomas," BMC cancer, vol. 11, no. 1, p. 198, 2011.

[24] G. William and J. Kaelin, "The von Hippel-Lindau protein, HIF hydroxylation, and oxygen sensing," Biochemical and Biophysical Research Communications, vol. 338, no. 1, pp. 627638, 2005.

[25] C. C. L. Wong, A. K. L. Kai, and I. O. L. Ng, "The impact of hypoxia in hepatocellular carcinoma metastasis," Frontiers in Medicine, vol. 8, no. 1, pp. 33-41, 2014. 
[26] C. Haan, G. Walbrecq, I. Kozar, I. Behrmann, and A. D. Zimmer, "Phosphorylation of the pyruvate dehydrogenase complex precedes HIF-1-mediated effects and pyruvate dehydrogenase kinase 1 upregulation during the first hours of hypoxic treatment in hepatocellular carcinoma cells," Hypoxia (Auckl), vol. 4, pp. 135-145, 2016.

[27] R. K. Lai, I. M. Xu, D. K. Chiu et al., "NDUFA4L2 fine-tunes oxidative stress in hepatocellular carcinoma," Clinical Cancer Research, vol. 22, no. 12, pp. 3105-3117, 2016.

[28] R. Liu, Y. Li, L. Tian et al., "Gankyrin drives metabolic reprogramming to promote tumorigenesis, metastasis and drug resistance through activating $\beta$-catenin/c-Myc signaling in human hepatocellular carcinoma," Cancer Letters, vol. 443, pp. 34-46, 2019.

[29] J. H. Kim, H. Y. Kim, Y. K. Lee et al., "Involvement of mitophagy in oncogenic K-Ras-induced transformation: overcoming a cellular energy deficit from glucose deficiency," Autophagy, vol. 7, no. 10, pp. 1187-1198, 2011.

[30] J. Kim, L. Yu, W. Chen et al., "Wild-type p53 promotes cancer metabolic switch by inducing PUMA-dependent suppression of oxidative phosphorylation," Cancer Cell, vol. 35, no. 2, pp. 191-203.e8, 2019.

[31] V. J. N. Bykov, S. E. Eriksson, J. Bianchi, and K. G. Wiman, "Targeting mutant p53 for efficient cancer therapy," Nature Reviews. Cancer, vol. 18, no. 2, pp. 89-102, 2018.

[32] P. H. C. Diniz, S. D. C. Silva, P. V. T. Vidigal et al., "Expression of MAPK and PI3K/AKT/mTOR proteins according to the chronic liver disease etiology in hepatocellular carcinoma," Journal of Oncology, vol. 2020, Article ID 4609360, 9 pages, 2020.

[33] X. Yang, M. Gao, M. Miao et al., "Combining combretastatin A4 phosphate with ginsenoside Rd synergistically inhibited hepatocellular carcinoma by reducing HIF- $1 \alpha$ via PI3K/AKT/mTOR signalling pathway," The Journal of Pharmacy and Pharmacology, vol. 73, no. 2, pp. 263-271, 2021.

[34] L. Yu, X. Chen, L. Wang, and S. Chen, "The sweet trap in tumors: aerobic glycolysis and potential targets for therapy," Oncotarget, vol. 7, no. 25, pp. 38908-38926, 2016.

[35] X. Chen, L. Wang, and H. Wang, "LINC01638 lncRNA promotes cancer cell proliferation in hepatocellular carcinoma by increasing cancer cell glucose uptake," Oncology Letters, vol. 18, no. 4, pp. 3811-3816, 2019.

[36] S. Wei, Q. Fan, L. Yang et al., "Promotion of glycolysis by HOTAIR through GLUT1 upregulation via mTOR signaling," Oncology Reports, vol. 38, no. 3, pp. 1902-1908, 2017.

[37] X. Zhuang, Y. Chen, Z. Wu et al., "Mitochondrial miR-181a$5 \mathrm{p}$ promotes glucose metabolism reprogramming in liver cancer by regulating the electron transport chain," Carcinogenesis, vol. 41, no. 7, pp. 972-983, 2020.

[38] L. Ren, Y. Yao, Y. Wang, and S. Wang, "MiR-505 suppressed the growth of hepatocellular carcinoma cells via targeting IGF-1R," Bioscience reports, vol. 39, no. 7, 2019.

[39] R. Shang, M. Wang, B. Dai et al., "Long noncoding RNASLC2A1-AS1 regulates aerobic glycolysis and progression in hepatocellular carcinoma via inhibiting the STAT3/FOXM1/GLUT1 pathway," Molecular Oncology, vol. 14, no. 6, pp. 1381-1396, 2020.

[40] W. Liu, L. Kang, J. Han et al., "miR-342-3p suppresses hepatocellular carcinoma proliferation through inhibition of IGF-1Rmediated Warburg effect," Oncotargets and Therapy, vol. 11, pp. 1643-1653, 2018.
[41] Y. Hu, Z. Yang, D. Bao, J. S. Ni, and J. Lou, "miR-455-5p suppresses hepatocellular carcinoma cell growth and invasion via IGF-1R/AKT/GLUT1 pathway by targeting IGF-1R," Pathology-Research and Practice, vol. 215, no. 12, article 152674, 2019.

[42] W. Yi, M. J. Tu, Z. Liu et al., "Bioengineered miR-328-3p modulates GLUT1-mediated glucose uptake and metabolism to exert synergistic antiproliferative effects with chemotherapeutics," Acta Pharmaceutica Sinica B, vol. 10, no. 1, pp. 159-170, 2020.

[43] Y. Lai, H. Huang, M. Abudoureyimu et al., "Non-coding RNAs: emerging regulators of glucose metabolism in hepatocellular carcinoma," American Journal of Cancer Research, vol. 10, no. 12, pp. 4066-4084, 2020.

[44] L. Song, W. Zhang, Z. Chang et al., "miR-4417 targets tripartite motif-containing 35 (TRIM35) and regulates pyruvate kinase muscle 2 (PKM2) phosphorylation to promote proliferation and suppress apoptosis in hepatocellular carcinoma cells," Medical Science Monitor, vol. 23, article 900296, pp. 17411750, 2017.

[45] Q. Li, X. Pan, D. Zhu, Z. Deng, R. Jiang, and X. Wang, "Circular RNA MAT2B promotes glycolysis and malignancy of hepatocellular carcinoma through the miR-338-3p/PKM2 Axis under hypoxic stress," Hepatology, vol. 70, no. 4, pp. 12981316, 2019.

[46] Y. F. Guan, Q. L. Huang, Y. L. Ai et al., "Nur77-activated lncRNA WFDC21P attenuates hepatocarcinogenesis via modulating glycolysis," Oncogene, vol. 39, no. 11, pp. 2408-2423, 2020.

[47] L. F. Zhang, J. T. Lou, M. H. Lu et al., "Suppression of miR199a maturation by HuR is crucial for hypoxia-induced glycolytic switch in hepatocellular carcinoma," The EMBO journal, vol. 34, no. 21, pp. 2671-2685, 2015.

[48] Q. Xu, C. Dou, X. Liu et al., "Oviductus ranae protein hydrolysate $(\mathrm{ORPH})$ inhibits the growth, metastasis and glycolysis of HCC by targeting miR-491-5p/PKM2 axis," Biomedicine \& Pharmacotherapy, vol. 107, pp. 1692-1704, 2018.

[49] A. M. Liu, Z. Xu, F. H. Shek et al., "miR-122 targets pyruvate kinase M2 and affects metabolism of hepatocellular carcinoma," PLoS One, vol. 9, no. 1, article e86872, 2014.

[50] Y. Zhao, L. Zhou, H. Li et al., "Nuclear-Encoded lncRNA MALAT1 Epigenetically Controls Metabolic Reprogramming in HCC Cells through the Mitophagy Pathway," Molecular Therapy-Nucleic Acids, vol. 23, pp. 264-276, 2021.

[51] J. H. Lee, D. H. Shin, W. Y. Park et al., "IDH1 R132C mutation is detected in clear cell hepatocellular carcinoma by pyrosequencing," World journal of surgical oncology, vol. 15, no. 1, p. 82, 2017.

[52] S. Chang, S. Yim, and H. Park, "The cancer driver genes IDH1/2, JARID1C/ KDM5C, and UTX/ KDM6A: crosstalk between histone demethylation and hypoxic reprogramming in cancer metabolism," Experimental \& Molecular Medicine, vol. 51, no. 6, pp. 1-17, 2019.

[53] R. Domenis, M. Comelli, E. Bisetto, and I. Mavelli, "Mitochondrial bioenergetic profile and responses to metabolic inhibition in human hepatocarcinoma cell lines with distinct differentiation characteristics," Journal of Bioenergetics and Biomembranes, vol. 43, no. 5, article 9380, pp. 493-505, 2011.

[54] D. DeWaal, V. Nogueira, A. R. Terry et al., "Hexokinase-2 depletion inhibits glycolysis and induces oxidative phosphorylation in hepatocellular carcinoma and sensitizes to metformin," Nature communications, vol. 9, no. 1, p. 446, 2018. 
[55] A. S. Tan, J. W. Baty, L. F. Dong et al., "Mitochondrial genome acquisition restores respiratory function and tumorigenic potential of cancer cells without mitochondrial DNA," Cell Metabolism, vol. 21, no. 1, pp. 81-94, 2015.

[56] F. Jin, Y. Wang, Y. Zhu et al., "The miR-125a/HK2 axis regulates cancer cell energy metabolism reprogramming in hepatocellular carcinoma," Scientific Reports, vol. 7, no. 1, p. 3089, 2017.

[57] J. W. Locasale and L. C. Cantley, "Altered metabolism in cancer," BMC biology, vol. 8, no. 1, 2010.

[58] C. Dou, H. Mo, T. Chen et al., "ZMYND8 promotes the growth and metastasis of hepatocellular carcinoma by promoting HK2-mediated glycolysis," Pathology - Research and Practice, vol. 219, article id 153345, 2021.

[59] X. Huang, G. Gan, X. Wang, T. Xu, and W. Xie, “The HGFMET axis coordinates liver cancer metabolism and autophagy for chemotherapeutic resistance," Autophagy, vol. 15, no. 7, pp. 1258-1279, 2019.

[60] M. Yang, J. Li, P. Gu, and X. Fan, "The application of nanoparticles in cancer immunotherapy: Targeting tumor microenvironment," Bioactive materials, vol. 6, no. 7, pp. 1973-1987, 2020.

[61] A. Brand, K. Singer, G. E. Koehl et al., "LDHA-associated lactic acid production blunts tumor immunosurveillance by $\mathrm{T}$ and NK cells," Cell Metabolism, vol. 24, no. 5, pp. 657-671, 2016.

[62] U. E. Martinez-Outschoorn, Z. Lin, C. Trimmer et al., "Cancer cells metabolically "fertilize" the tumor microenvironment with hydrogen peroxide, driving the Warburg effect," Cell Cycle, vol. 10, no. 15, pp. 2504-2520, 2011.

[63] X. Y. Hu and L. B. Ivashkiv, "Cross-regulation of signaling pathways by interferon- $\gamma$ : implications for immune responses and autoimmune diseases," Immunity, vol. 31, no. 4, pp. 539550, 2009.

[64] L. J. Reitzer, B. M. Wice, and D. Kennell, "Evidence that glutamine, not sugar, is the major energy source for cultured HeLa cells.," The Journal of Biological Chemistry, vol. 254, no. 8, pp. 2669-2676, 1979.

[65] X. S. Chen, L. Y. Li, Y. D. Guan, J. M. Yang, and Y. Cheng, "Anticancer strategies based on the metabolic profile of tumor cells: therapeutic targeting of the Warburg effect," Acta Pharmacologica Sinica, vol. 37, no. 8, pp. 1013-1019, 2016.

[66] Y. Sugiyama, T. Shudo, S. Hosokawa, A. Watanabe, M. Nakano, and A. Kakizuka, "Emodin, as a mitochondrial uncoupler, induces strong decreases in adenosine triphosphate (ATP) levels and proliferation of B16F10 cells, owing to their poor glycolytic reserve," Genes Cells, vol. 24, no. 8, pp. 569584, 2019.

[67] J. Calderaro, M. Ziol, V. Paradis, and J. Zucman-Rossi, "Molecular and histological correlations in liver cancer," Journal of Hepatology, vol. 71, no. 3, pp. 616-630, 2019.

[68] Z. F. Karagonlar, S. Akbari, M. Karabicici et al., "A Novel Function for KLF4 in Modulating the De-differentiation of EpCAM-/CD133- nonStem Cells into EpCAM+/CD133+ Liver Cancer Stem Cells in HCC Cell Line HuH7," Cells, vol. 9, no. 5, p. 1198, 2020.

[69] R. Shang, M. Wang, B. Dai et al., "Long noncoding RNA SLC2A1-AS1 regulates aerobic glycolysis and progression in hepatocellular carcinoma via inhibiting the STAT3/FOXM1/GLUT1 pathway," Molecular Oncology, vol. 14, no. 6, pp. 1381-1396, 2020.
[70] A. C. K. Lee, P. M. Lau, Y. W. Kwan, and S. K. Kong, "Mitochondrial fuel dependence on glutamine drives chemoresistance in the cancer stem cells of hepatocellular carcinoma," International Journal of Molecular Sciences, vol. 22, no. 7, p. 3315, 2021.

[71] H. Hu, X. Ding, Y. Yang et al., "Changes in glucose-6phosphate dehydrogenase expression results in altered behavior of HBV-associated liver cancer cells," American Journal of Physiology. Gastrointestinal and Liver Physiology, vol. 307, no. 6, pp. G611-G622, 2014.

[72] M. C. Herzig, J. A. Zavadil, K. Street et al., "DNA Alkylating Agent Protects Against Spontaneous Hepatocellular Carcinoma Regardless of O6-Methylguanine-DNA Methyltransferase Status," Cancer Prevention Research, vol. 9, no. 3, pp. 245252, 2016.

[73] J. Sun, J. Li, Z. Guo et al., "Overexpression of pyruvate dehydrogenase E1 $\alpha$ subunit inhibits Warburg effect and induces cell apoptosis through mitochondria-mediated pathway in hepatocellular carcinoma," Oncology Research, vol. 27, no. 4, pp. 407-414, 2019.

[74] E. Dapat, S. Jacinto, and T. Efferth, "Substrate specificity of Aglaia loheri active isolate towards P-glycoprotein in multidrug-resistant cancer cells," Natural Product Communications, vol. 11, no. 11, pp. 1683-1688, 2016. 